\title{
Signs of warm water on Saturn's moon
}

Astrobiologists have welcomed the news that Saturn's small moon Enceladus has a huge geyser thatspews water vapour and dust thousands of kilometres into space. Itmeans the satellite may be hiding one of the most accessible reservoirs of liquid water in the Solar System.

Evidence for the geyser was discovered by NASA's Cas sini probe during three fly-bys in 2005. "Cassini essentially flew through it," says planetary scientist Jonathan Lunine of the University of Arizona, Tucson.

According to a suite of papers in last week's Science (see Science 311, 1388; 2006), the plume, which also contains carbon dioxide, methane, nitrogen and propane, is probably fed by a reservoir of liquid water thatnestles just beneath the icy surface.

The idea that material could be welling up beneath the surface of Enceladus isn't new. When the Voyager probes flew past in 1980 and 1981, they spotted a flat area around the south pole that looked as though it had recently been resurfaced by volcanic activity; there was no sign of craters left by meteorite impacts, suggesting they had been wiped away. Astronomers also speculatedfrom Voyager data that Saturn's Ering of fine particles was being fed by material from Enceladus.

The latestobservations support that idea, and suggest that the geysercould in part be driven by heat from the movement of Enceladus' insides, which slosh around as the moon is pulled back and forth by its neighbours' gravity. Heating from the decay of radioactive elementsalso contributes to the process. The Cassiniteam estimates that at least 150 kilograms of material a second comes from the plume, which is more than enoughto supply Saturn's Ering.

But the probe did not find any ammonia - previously suggested as the antifreeze that helps keep internal water liquid in this chilly part of the Solar System. So parts of
Enceladus may be much warmer than anyone expected, a possibility that has excited astrobiologists.

"We have found another environmentin our Solar System, in a very surprising place, that could host living organisms," says Caroline Porco of the Space Science Institute in Boulder, Colorado, who leads Cassini's imaging team. Thatmakes Enceladusan attractive place to explore, says Lunine. The icecoveredoceans of Jupiter's large moon Europa remains the main target of astrobiologists' interest. But its frozen crust may be kilometres thick. "What's different here is that pockets of liquid water may be no more than tens of metres below the surface," says Andrew Ingersoll, an atmospheric scientist at the California Institute of Technology, Pasadena. Cassini's nextclose fly-by in spring 2008 should help to find out for sure.

Mark Peplow

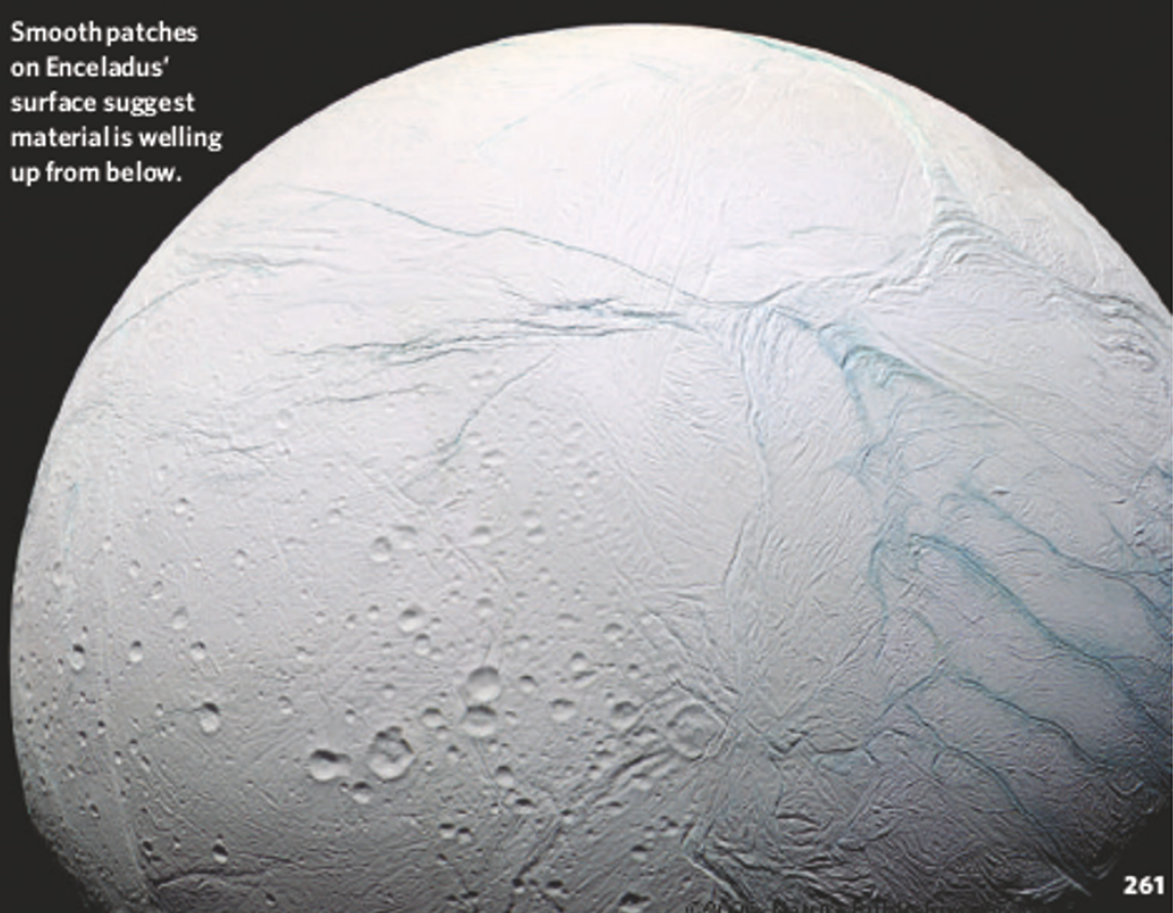

